\title{
Clinical analysis of a patient with a benign lesion of the pharynx misdiagnosed as functional thyroid cancer metastasis
}

\author{
RENFEI WANG, JIAN TAN, GUIZHI ZHANG and QIANG JIA \\ Department of Nuclear Medicine, Tianjin Medical University General Hospital, Tianjin 300052, P.R. China \\ Received June 23, 2014; Accepted February 10, 2015
}

DOI: $10.3892 /$ etm.2015.2335

\begin{abstract}
Papillary thyroid carcinoma (PTC) usually has a lower malignancy rate and better prognosis than other types of thyroid cancer. It tends to spread through the lymphatic system, and the percentage of lymph node metastasis to the neck is $50-70 \%$ in patients at the time of diagnosis. Iodine-131 $\left({ }^{131} \mathrm{I}\right)$ whole-body scanning is very valuable in the diagnosis and positioning of functional metastatic lesions. However, a variety of comprehensive test results are frequently required to make an appropriate judgment. The present case report describes the case of a patient with PTC where a benign lesion of the pharynx was misdiagnosed as functional metastasis. Some remnant thyroid tissue and an abnormal accumulation of ${ }^{131} \mathrm{I}$ in the oropharynx and at the right side of the neck were observed by ${ }^{131}$ I whole-body scanning one week after the first therapeutic dose. Following the second therapeutic dose, quasi-circular trapping of ${ }^{131} \mathrm{I}$ by the pharynx persisted. Magnetic resonance imaging enhanced scanning of the oropharynx showed markedly uneven enhancement inside the lesion, and it was considered as a metastatic lesion. However, the serological test results of the patient revealed that there was no increase in the thyroglobulin level and did not support the diagnosis of progressive disease. Therefore, a biopsy on the lesion was conducted under local anesthesia. The histopathological examination exhibited salivary gland tissue and chronic inflammation. Thus, it was necessary to analyze the imaging results, as well as serologic testing outcomes and pathological findings comprehensively, in order to make the correct diagnosis.
\end{abstract}

\section{Introduction}

Thyroid cancer is the most common malignant tumor of the endocrine system, accounting for $<1 \%$ of all carcinomas (1).

Correspondence to: Professor Jian Tan, Department of Nuclear Medicine, Tianjin Medical University General Hospital, 154 Anshan Road, Heping, Tianjin 300052, P.R. China

E-mail: tanpost@163.com

Key words: papillary thyroid carcinoma, functional metastasis, iodine-131 whole-body scanning, false positive
Thyroid carcinomas are derived from two cell types presenting in the thyroid gland. Among them, papillary and follicular thyroid carcinomas (PTC and FTC), arising from follicular cells, are also termed as differentiated thyroid carcinoma (DTC) due to their higher differentiation degree and iodine-uptake ability. The incidence of thyroid cancer, particularly PTC, is increasing worldwide (2). PTC is the most common form of thyroid cancer, accounting for $75-80 \%$ of thyroid carcinomas (1) with a $1 \%$ mortality rate and almost $5 \%$ recurrence rate (3). PTC has a lower malignancy rate and better prognosis than other types of thyroid cancer. It tends to spread through the lymphatic system, and the percentage of lymph node metastasis to the neck is $50-70 \%$ in patients at the time of diagnosis (3). Distant metastasis, such as in the lung and bone, is uncommon; however, if it is present, the prognosis is poor. Iodine-131 $\left({ }^{131} \mathrm{I}\right)$ whole-body scanning is very valuable not only in the diagnosis and positioning of functional metastatic lesions (4), but also in the determination of treatment strategies and prognosis assessment (5).

The present case report describes a patient with PTC where a benign lesion of the pharynx was misdiagnosed as functional metastasis. Written informed consent was obtained from the patient for publication of this case study. The study was approved by the ethics committee of Tianjin Medical University General Hospital (Tianjin, China) and adhered to the tenets of the Declaration of Helsinki.

\section{Case report}

A 44-year-old male was admitted to the radionuclide treatment ward of Tianjin Medical University General Hospital to accept a third ${ }^{131} \mathrm{I}$ therapy. The patient had suffered sleep apnea syndrome for $>10$ years and he had smoked for $>20$ years, 10 to 20 cigarettes every day. The patient had a remnant neck mass that had been present for 2 years following a near-total thyroidectomy with dissection of lateral lymph node compartments that yielded a $22 \mathrm{~mm}$ diameter, non-encapsulated PTC on the left lobe. Lymph node metastasis was diagnosed in the left side of the neck one year ago. A preoperative neck computed tomography (CT) scan revealed thickening of the oropharyngeal soft tissue.

The first therapeutic dose $(3.7 \mathrm{GBq})$ of ${ }^{131} \mathrm{I}$ was administered 2 months following the surgery. Laboratory findings for the patient are presented in Table I. Whole-body scanning performed with ${ }^{131} \mathrm{I} 1$ week after administration detected some 
Table I. Biological data at each time-point of therapeutic ${ }^{131}$ I administration.

\begin{tabular}{lcccccr}
\hline $\begin{array}{l}\text { Treatment } \\
\text { no. }\end{array}$ & $\begin{array}{c}{ }^{131} \mathrm{I} \text { dose } \\
\mathrm{GBq}\end{array}$ & $\begin{array}{c}\mathrm{Free}_{3}{ }^{\mathrm{a}} \\
(\mathrm{pmol} / \mathrm{l})\end{array}$ & $\begin{array}{c}\text { Free T}_{4}{ }^{\mathrm{b}} \\
(\mathrm{pmol} / \mathrm{l})\end{array}$ & $\begin{array}{c}\mathrm{TSH}^{\mathrm{c}} \\
(\mu \mathrm{IU} / \mathrm{ml})\end{array}$ & $\begin{array}{c}\mathrm{Tg}^{\mathrm{d}} \\
(\mathrm{ng} / \mathrm{ml})\end{array}$ & $\begin{array}{r}\mathrm{TgAb}^{\mathrm{e}} \\
(\mathrm{IU} / \mathrm{ml})\end{array}$ \\
\hline 1 & 3.7 & 3.05 & 7.94 & 45.94 & 6.73 & 23.6 \\
2 & 3.7 & 2.36 & 6.07 & 50.52 & 6.80 & $<20.0$ \\
3 & 3.7 & 2.25 & 6.99 & 56.03 & 4.33 & $<20.0$ \\
\hline
\end{tabular}

${ }^{\mathrm{a}}$ Normal range, 3.5-6.5 pmol/1; ${ }^{\mathrm{b}}$ normal range, 11.5-23.5 pmol/1; ${ }^{\mathrm{c}}$ normal range, 0.3-5.0 $\mu \mathrm{IU} / \mathrm{ml}$; ${ }^{\mathrm{d}}$ normal range, 0-40 ng/ml; ${ }^{\mathrm{e}} \mathrm{normal} \mathrm{range}$, 0-55 IU/ml. T3, triiodothyronine; T4, thyroxine; TSH, thyroid-stimulating hormone; Tg, thyroglobulin; TgAb, Tg antibody.

A

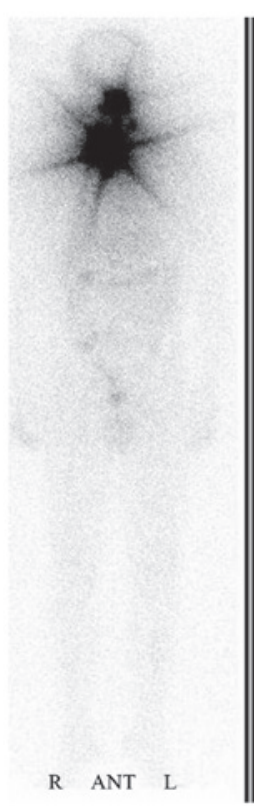

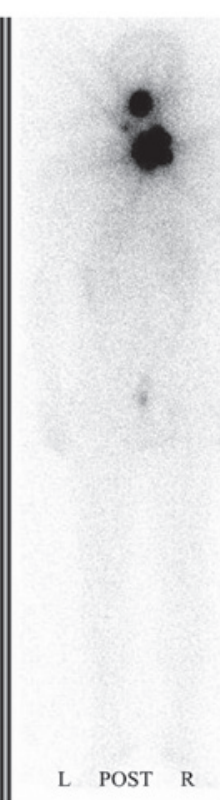

B
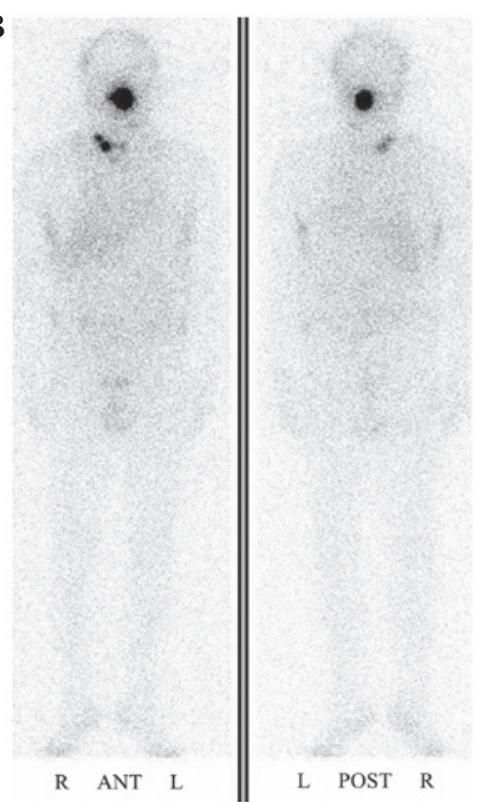

Figure $1 .{ }^{131}$ I whole-body scanning. (A) One week after the first therapeutic dose, some remnant thyroid tissue and an abnormal accumulation of ${ }^{131}$ I in the oropharynx and right side of neck were observed. (B) Five days after the second therapeutic dose, foci for ${ }^{131}$ I uptake in the oropharynx and right lateral neck were observed. Quasi-circular trapping of ${ }^{131} \mathrm{I}$ by the pharynx persisted. R, right; L, left; ANT, anterior; POST, posterior.

remnant thyroid tissue, and an abnormal accumulation of ${ }^{131} \mathrm{I}$ in the oropharynx and right side of the neck, which could not exclude the diagnosis of lymph node metastasis (Fig. 1A). Thyroid hormone replacement with levothyroxine was initiated. The second therapeutic dose $(3.7 \mathrm{GBq})$ of ${ }^{131} \mathrm{I}$ was administered 4 months later. Scintigraphy performed 5 days after the second ${ }^{131} \mathrm{I}$ administration revealed the persistence of ${ }^{131} \mathrm{I}$ accumulation in the oropharyngeal focus and right lateral neck (Fig. 1B). ${ }^{131}$ I scanning revealed that the residual thyroid tissue had almost disappeared, indicating that the remnant ablation had succeeded; however, quasi-circular trapping of ${ }^{131}$ I by the pharynx persisted.

The patient stopped using levothyroxine two weeks prior to admission for the third ${ }^{131} \mathrm{I}$ therapy, and the blood level of thyroid-stimulating hormone (TSH) was $56.03 \mu \mathrm{IU} / \mathrm{ml}$. The levels of thyroglobulin ( $\mathrm{Tg}$ ) and $\mathrm{Tg}$ antibody $(\mathrm{Tg} \mathrm{Ab})$ were $4.33 \mathrm{ng} / \mathrm{ml}$ and $<20.0 \mathrm{IU} / \mathrm{ml}$ respectively. The $\mathrm{Tg}$ level prior to the first and second treatments was 6.73 and $6.80 \mathrm{ng} / \mathrm{ml}$, respectively (Table I). Four months following the second treatement, the patient agreed to accept a third ${ }^{131} \mathrm{I}$ therapy. The patient was admitted to hospital for further diagnosis; an examination by magnetic resonance imaging (MRI) plain and enhanced scanning of the oropharynx was conducted. The results were as follows: An oval long T1/T2 signal lesion was observed in the left parapharyngeal space; there were multiple nodules within the lesion, the size of which was $4.1 \times 3.5 \times 2.2 \mathrm{~cm}^{3}$. The upper border of the lesion was close to the top of the nasopharynx; the lower boundary was near the bottom of the oropharyngeal cavity and the outer border was inside the medial pterygoid. The left sidewall of the nasopharyngeal and oropharyngeal cavity was compressed and moved to the right. The nasopharyngeal and oropharyngeal cavity had narrowed. The boundary between the lesion and the left sidewall of the nasopharyngeal and oropharyngeal cavity was not clear. MRI enhanced scanning suggested that the lesion exhibited markedly uneven enhancement on the left side of the pharynx, and an enhanced nodule and patchy non-enhanced area were visible inside the lesion (Fig. 2). Following consideration of the medical history of the patient, the possibility of the lesion being a metastatic lesion was considered, and indicated that further examination was necessary. Nasopharyngeal endoscopy revealed that the nasal septum was deviated, there was a nodular mass at the rear end of the left septum, the properties of the nasopharyngeal neoplasm were unclear, and 

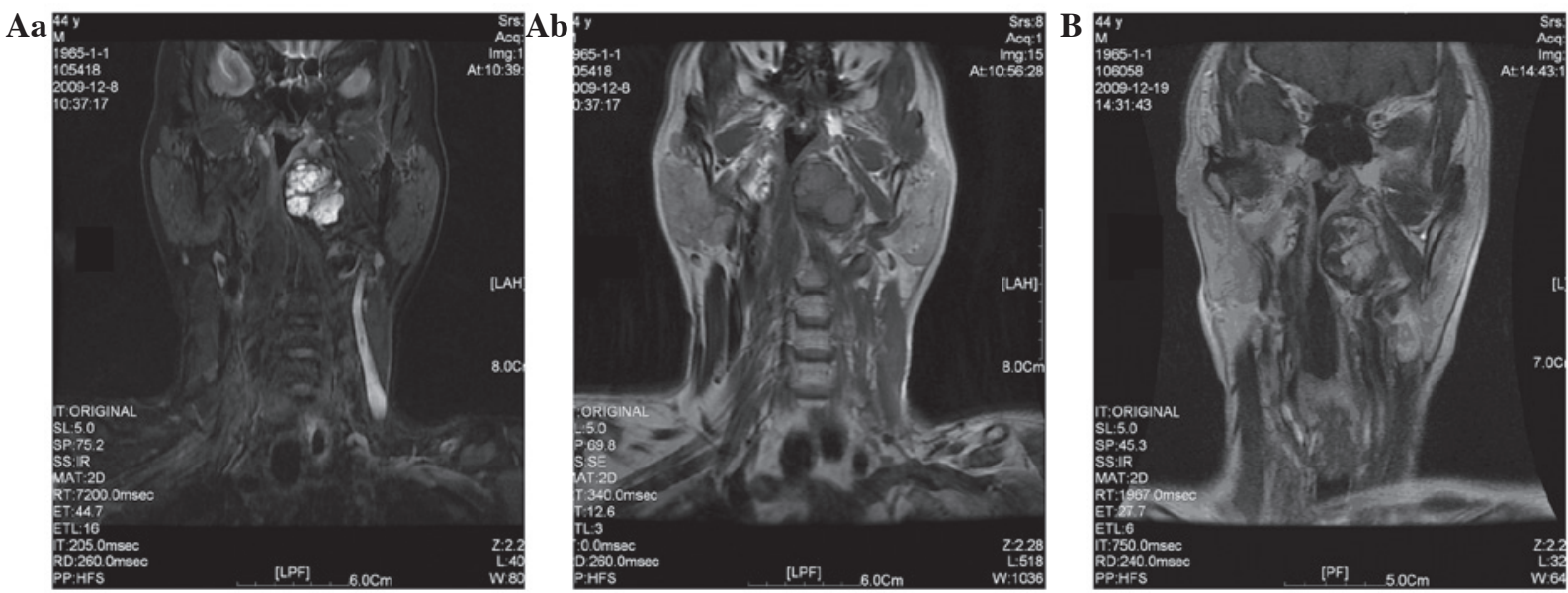

Figure 2. (A) In MRI plain scanning of the oropharynx, an oval long T1 (Aa)/T2 (Ab)-weighted signal lesion was visible in the left parapharyngeal space, and there were multiple nodules within the lesion. The left side wall of the nasopharyngeal and oropharyngeal cavity was compressed and moved to the right. (B) In MRI enhanced scanning of the oropharynx, the lesion exhibited evidently uneven enhancement, and an enhanced nodule and patchy non-enhanced area could also be seen inside the lesion. MRI, magnetic resonance imaging.

\section{A}

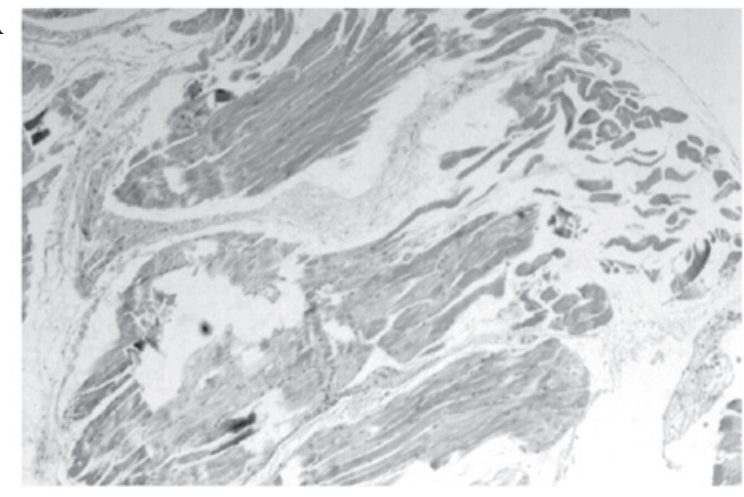

B

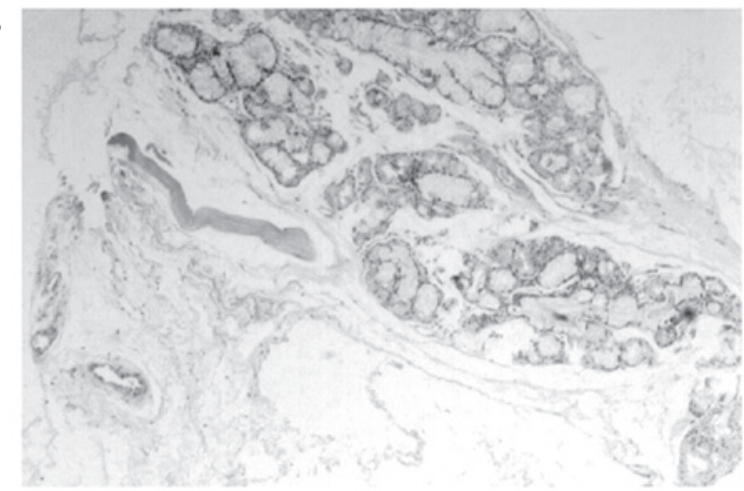

Figure 3. Pathology of the lesion in the left parapharyngeal space revealed (A) skeletal muscle cells along with fibrosis and (B) salivary gland tissue. Magnification, $\mathrm{x} 40$.

the nasopharyngeal cavity and nasopharyngeal groove were narrowed, suggesting that a biopsy was required. A biopsy was then conducted on the left side of the pharynx under local anesthesia. The pathological examination revealed that there were a large number of skeletal muscle cells along with fibrosis in the muscles, salivary gland tissue was found in the muscle, and the small pieces of mucosal tissue present in the covering epithelium exhibited chronic inflammation (Fig. 3). When the wound was healed, the patient was given the third therapeutic dose (3.7 GBq) of ${ }^{131} \mathrm{I}$. Whole-body scanning with a therapeutic dose of ${ }^{131} \mathrm{I}$ demonstrated that the abnormal accumulation of ${ }^{131} \mathrm{I}$ in the right side of the neck had faded, the abnormal accumulation in the oropharyngeal cavity had reduced in size, and the degree of accumulation was unchanged.

\section{Discussion}

Total or near-total thyroidectomy, adjuvant radioactive iodine therapy and TSH suppression therapy with levothyroxine are recognized as the best comprehensive treatment protocols for patients with DTC (6). The treatment of DTC and the diagnosis of its metastases are based on the capacity of thyroid cells to actively trap radioactive ${ }^{131} \mathrm{I}(7) .{ }^{131} \mathrm{I}$ whole-body scanning is very valuable, not only in the selection of the treatment strategy and appropriate therapeutic dosage but also in follow-up visits and for determining recurrence and metastasis risks effectively $(8,9)$. However, ${ }^{131}$ I whole-body scanning may present some false positive results, the reasons being physiological uptake, pathological accumulation and the retention of secretions (10).

Two therapeutic doses of ${ }^{131} \mathrm{I}$ were given to the patient on the second and sixth months after surgery. Levothyroxine was administered to the patient to suppress the TSH level regularly between doses. The first of these two whole-body scans with a therapeutic dose of ${ }^{131}$ I demonstrated an abnormal accumulation in the oropharynx, which was considered as a metastatic lesion. However, the serological test results of the patient, which showed that the Tg level did not rise, did not support the diagnosis of progressive disease. Therefore, an MRI scan of the oropharynx was arranged for this patient in order to make a definitive diagnosis. The scan revealed a space-occupying lesion in the left parapharyngeal space, which was unevenly enhanced in the enhanced MRI scan, and the possibility that the lesion was a metastatic lesion was considered when the medical history of the patient with regard to the tumor was considered. The histopathological examination of the biopsy tissue confirmed that the abnormal ${ }^{131} \mathrm{I}$-accumulating mass was benign. The main reason of this false positive result may be physiological uptake by the salivary gland tissue present in the lesion. Furthermore, the 
pathological accumulation of ${ }^{131} \mathrm{I}$ in the lesion may be connected with chronic inflammation (11). In addition, a certain degree of radiation injury took place in the benign mass, with pathological changes including fibrous connective tissue hyperplasia in skeletal muscle cells (12), showing that the lesion presented a certain degree of degeneration.

At present, there is no examination considered as the gold standard for the diagnosis of the recurrence and metastasis of DTC. The authors of the present study believe that a combination of ${ }^{131} \mathrm{I}$ whole-body scanning, ${ }^{18} \mathrm{~F}$-fluorodeoxyglucose positron emission tomography, CT and MRI is the optimal method for the detection of recurrent and metastatic disease. It is necessary to analyze the imaging results, as well as serologic testing outcomes and pathological findings comprehensively, in order to make the correct diagnosis as early as possible and to enable patients to be treated properly with individualized strategies.

\section{References}

1. Cobin RH, Gharib H, Bergman DA, et al; Thyroid Carcinoma Task Force: AACE/AAES medical/surgical guidelines for clinical practice: management of thyroid carcinoma. American Association of Clinical Endocrinologists. American College of Endocrinology. Endocr Prac 7: 202-220, 2001

2. Al-Humadi H, Zarrros A, Al-Saigh R and Liapi C: Genetic basis and gene therapy trials for thyroid cancer. Cancer Genomics Proteomics 7: 31-49, 2010.

3. Hay ID: Papillary thyroid carcinoma. Endocrinol Metab Clin North Am 19: 545-576, 1990.
4. Sarkar SD, Kalapparambath TP and Palestro CJ: Comparison of 123I and 131I for whole-body imaging in thyroid cancer. J Nucl Med 5: 632-634, 2002.

5. Grewal RK, Tuttle RM, Fox J, et al: The effect of posttherapy 131I SPECT/CT on risk classification and management of patients with differentiated thyroid cancer. J Nucl Med 9: 1361-1367, 2010.

6. Chinese Society of Endocrinology, Endocrine Group of Surgery Branch of Chinese Medical Association, Committee for Head and Neck Oncology of Chinese Anti-cancer Association, Society of Nuclear Medicine of Chinese Medical Association: Clinical guidelines for the diagnosis and management of thyroid nodules and differentiated thyroid cancer. Zhonghua He Yi Xue Yu Fen Zi Ying Xiang Za Zhi 33: 96-115, 2013 (In Chinese)

7. Leger AF, Pellan M, Dagousset F, et al: A case of stunning of lung and bone metastases of papillary thyroid cancer after a therapeutic dose $(3.7 \mathrm{GBq})$ of $131 \mathrm{I}$ and review of the literature: implications for sequential treatments. Br J Radiol 78: 428-432, 2005.

8. Lind P and Kohlfürst S: Respective roles of thyroglobulin, radioiodine imaging and positron emission tomography in the assessment of thyroid cancer. Semin Nucl Med 36: 194-205, 2006.

9. Spanu A, Solinas ME, Chessa F, et al: 131I SPECT/CT in the follow-up of differentiated thyroid carcinoma: incremental value versus planar imaging. J Nucl Med 50: 184-190, 2009.

10. Mitchell G, Pratt BE, Vini L, et al: False positive 131I whole body scans in thyroid cancer. Br J Radiol 73: 627-635, 2000.

11. Ma C, Kuang A, Xie JW and Ma TK: Possible explanations for patients with discordant findings of serum thyroglobulin and 131I whole-body scanning. J Nucl Med 46: 1473-1480, 2005.

12. Mandel L and Liu F: Salivary gland injury resulting from exposure to radioactive iodine: case reports. J Am Dent Assoc 138: 1582-1587, 2007. 